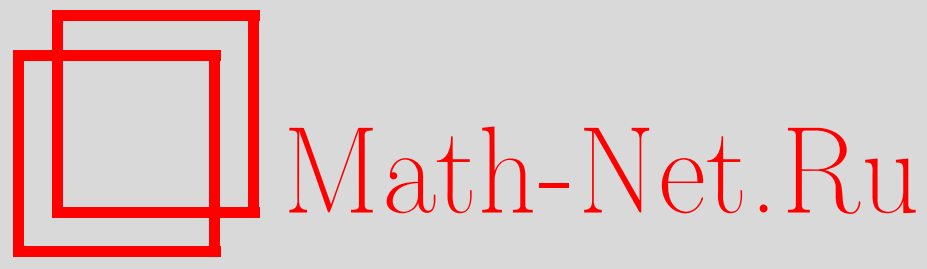

А. А. Саакян, М. С. Гиновян, О центральной предельной теореме для тёплицевых квадратичных форм от стационарных последовательностей, Теория вероятн. и ее примен., 2004, том 49, выпуск 4, 653-671

DOI: https://doi.org/10.4213/tvp187

Использование Общероссийского математического портала MathNet.Ru подразумевает, что вы прочитали и согласны с пользовательским соглашением

http://www.mathnet.ru/rus/agreement

Параметры загрузки:

IP: 107.22 .136 .117

26 апреля 2023 г., 16:52:02

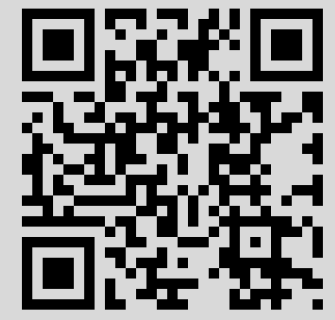




\section{О ЦЕНТРАЛЬНОЙ ПРЕДЕЛЬНОЙ ТЕОРЕМЕ ДЛЯ ТЁПЛИЦЕВЫХ КВАДРАТИЧНЫХ ФОРМ ОТ СТАЦИОНАРНЫХ ПОСЛЕДОВАТЕЛЬНОСТЕЙ ${ }^{1)}$}

Пусть $X(t), t=0, \pm 1, \pm 2, \ldots$, - вещественнозначная стационарная гауссовская последовательность со спектральной плотностью $f(\lambda)$. В статье рассматривается вопрос применимости центральной предельной теоремы (ЦПТ) для тёплицевой квадратичной формы $Q_{n}$ от переменных $X(t), t=1, \ldots, n$, порожденной некоторой интегрируемой четной функцией $g(\lambda)$. Предположив, что $f(\lambda)$ и $g(\lambda)$ - регулярно меняющиеся в точке $\lambda=0$ функции порядка $\alpha$ и $\beta$ соответственно, мы доказываем ЦПТ для стандартно нормированной квадратичной формы $Q_{n}$ в критическом случае $\alpha+\beta=\frac{1}{2}$.

Мы также показываем, что условие положительности и конечности асимптотической дисперсии квадратичной формы $Q_{n}$ не гарантирует выполнение ЦПТ для $Q_{n}$.

Ключевые слова и фразы: стационарная гауссовская последовательность, спектральная плотность, тёплицевы квадратичные формы, центральная предельная теорема, асимптотическая дисперсия, медленно меняющиеся функции.

1. Введение. Пусть $X(t), t=0, \pm 1, \pm 2, \ldots$, - центрированная $(\mathbf{E} X(t)=0)$ вещественнозначная стационарная гауссовская последовательность со спектральной плотностью $f(\lambda)$ и ковариационной функцией $r(t)$, т.е.

$$
r(t)=\int_{-\pi}^{\pi} e^{i \lambda t} f(\lambda) d \lambda .
$$

Мы рассматриваем вопрос об асимптотическом распределении (когда $n \rightarrow \infty)$ следующей тёплицевой квадратичной формы от процесса $X(t)$ :

$$
Q_{n}=\sum_{k, j=1}^{n} a(k-j) X(k) X(j),
$$

* Институт математики НАН Армении, пр. Маршала Баграмяна, 24-Б, 375019, Ереван, Армения; e-mail: mginovian@yahoo.com; sart@instmath.sci.am

1) Работа выполнена при финансовой поддержке грантов ANSEF (№PS58) и NFSAT/CRDF (№ MA 070-02/12011). 
где

$$
a(k)=\int_{-\pi}^{\pi} e^{i \lambda k} g(\lambda) d \lambda, \quad k=0, \pm 1, \pm 2, \ldots,
$$

суть коэффициенты Фурье некоторой вещественной, четной, интегрируемой функции $g(\lambda), \lambda \in \mathbb{T}=[-\pi, \pi]$. Функцию $g(\lambda)$ мы называем порождающей функцией квадратичной формы $Q_{n}$. Мы будем считать, что функции $f(\lambda)$ и $g(\lambda)$ являются $2 \pi$-периодичными.

Предельное распределение случайных величин (1.2) полностью определяется спектральной плотностью $f(\lambda)$ и порождающей функцией $g(\lambda)$, и, в зависимости от их свойств, оно может быть гауссовским (т.е. для $Q_{n}$ с подходящей нормировкой имеет место центральная предельная теорема) или не гауссовским.

Естественно рассмотреть следующие два вопроса.

а) При каких условиях на $f(\lambda)$ и $g(\lambda)$ предельное распределение $Q_{n}$ будет гауссовским?

b) Описать предельное распределение $Q_{n}$, если оно не гауссовское.

В данной статье мы изучаем по существу вопрос а). Эта проблема впервые была рассмотрена в классической монографии У. Гренандера и Г. Сегё [9] в качестве применения их теории об асимптотическом поведении следа произведения усеченных тёплицевых матриц.

Позже эта проблема была изучена И. А. Ибрагимовым [11] и М. Розенблатом [12] в связи со статистическими оценками для спектральной $(F(\lambda))$ и ковариационной $(r(t))$ функций соответственно. С 1986 г. интерес к вопросам a) и b) обновился в связи со статистическими выводами для сильно зависимых процессов (см., например, статьи Ф. Аврама [1], Р. Фокса и М. С. Такку [4], Л. Гирайтиса и Д. Сургайлиса [8], Н. Террин и М. С. Такку [14], М. Танигучи [17] и книгу М. Танигучи и Й. Какизавы [18]). В работах [1], [4] и [8] были получены достаточные условия для выполнения ЦПТ для квадратичной формы $Q_{n}$.

Для формулировки соответствующих результатов нам потребуется ряд обозначений. Обозначим через $\widetilde{Q}_{n}$ нормированную квадратичную форму:

$$
\widetilde{Q}_{n}=\frac{1}{\sqrt{n}}\left(Q_{n}-\mathbf{E} Q_{n}\right)
$$

Запись

$$
\widetilde{Q}_{n} \Longleftrightarrow N\left(0, \sigma^{2}\right)
$$

будет означать, что распределение случайной величины $\widetilde{Q}_{n}$ при $n \rightarrow \infty$ стремится к центрированному нормальному распределению с дисперсией $\sigma^{2}$. Через $T_{n}(f)$ и $T_{n}(g)$ мы обозначим тёплицевы матрицы размера $n \times n$, порожденные функциями $f$ и $g$ соответственно, т.е.

$$
T_{n}(f)=\|r(k-j)\|_{k, j=\overline{1, n}} \quad \text { и } \quad T_{n}(g)=\|a(k-j)\|_{k, j=\overline{1, n}}
$$


где $r(k)$ и $a(k)$ определены в $(1.1)$ и (1.3) соответственно. Через $C, M$, $C_{k}, M_{k}$ мы обозначим постоянные, которые могут быть разными в разных формулах.

Теорема А (Ф. Аврам). Пусть спектральная плотность $f(\lambda)$ u порождающая функиия $g(\lambda)$ таковь, ито $f(\lambda) \in L^{p_{1}}(\mathbb{T}), g(\lambda) \in L^{p_{2}}(\mathbb{T})$, где $p_{1}, p_{2} \geqslant 1$ u $1 / p_{1}+1 / p_{2} \leqslant \frac{1}{2}$. Тогда имеет место (1.5) $c$

$$
\sigma^{2}=16 \pi^{3} \int_{-\pi}^{\pi} f^{2}(\lambda) g^{2}(\lambda) d \lambda
$$

3 а м е ч а н и е 1.1. При $p_{1}=p_{2}=\infty$ теорема А была доказана У. Гренандером и Г. Сегё $[9$, теорема 11.6$])$, а в случае $p_{1}=2, p_{2}=\infty-$ И. А. Ибрагимовым [11] и М. Розенблатом [12].

Теорема В (Р. Фокс и М. С. Такку). Пусть выполнень следуюшие условия:

а) функции $f(\lambda)$ u $g(\lambda)$ непрерывны почти всюду и ограничены на $[\delta, \pi]$ при любом $\delta>0$;

b) существуют $\alpha<1$ и $\beta<1$ такие, что $\alpha+\beta<\frac{1}{2}$,

$$
\begin{array}{lll}
f(\lambda) \sim|\lambda|^{-\alpha} L_{1}(\lambda) & \text { при } & \lambda \rightarrow 0, \\
g(\lambda) \sim|\lambda|^{-\beta} L_{2}(\lambda) & \text { при } & \lambda \rightarrow 0,
\end{array}
$$

где $L_{1}(\lambda)$ и $L_{2}(\lambda)$ суть медленно меняюшиеся в точке $\lambda=0$ функиии.

Тогда имеет место (1.5) с дисперсией $\sigma^{2}$, определенной в (1.7).

Доказательства теорем А и В в [1] и [4] основаны на следующем хорошо известном представлении $k$-го кумулянта $\chi_{k}(\cdot)$ квадратичной формы $\widetilde{Q}_{n}$ (см., например, [9], [11]):

$$
\chi_{k}\left(\widetilde{Q}_{n}\right)=\left\{\begin{array}{lll}
0 & \text { при } & k=1, \\
n^{-k / 2} 2^{k-1}(k-1) ! \operatorname{tr}\left[T_{n}(f) T_{n}(g)\right]^{k} & \text { при } & k \geqslant 2,
\end{array}\right.
$$

где $\operatorname{tr}[A]-$ след матрицы $A$.

Другой подход, примененный в работе [8], позволил распространить теоремы A и В на линейные последовательности. В гауссовском случае этот результат может быть сформулирован следующим образом.

Теорема С (Л. Гирайтис и Д. Сургайлис). Предположим, что npu $n \rightarrow \infty$

$$
\chi_{2}\left(\widetilde{Q}_{n}\right)=\frac{2}{n} \operatorname{tr}\left[T_{n}(f) T_{n}(g)\right]^{2} \longrightarrow 16 \pi^{3} \int_{-\pi}^{\pi} f^{2}(\lambda) g^{2}(\lambda) d \lambda<\infty .
$$

Тогда имеет место (1.5), где дисперсия $\sigma^{2}$ определена в (1.7).

В работах [1] и [4] (см. также [8]) было установлено, что из условий каждой из теорем А и В вытекает (1.10). K сожалению, проверить выполнение условия (1.10) непросто. Более простое достаточное условие было найдено в [8]. 
Теорема D (Л. Гирайтис и Д. Сургайлис). Пусть $f \in L^{2}(\mathbb{T})$, $g \in L^{2}(\mathbb{T}), f g \in L^{2}(\mathbb{T}) u n p u \mu \rightarrow 0$

$$
\int_{-\pi}^{\pi} f^{2}(\lambda) g^{2}(\lambda-\mu) d \lambda \longrightarrow \int_{-\pi}^{\pi} f^{2}(\lambda) g^{2}(\lambda) d \lambda .
$$

Тогда имеет место (1.5), где дисперсия $\sigma^{2}$ определена в (1.7).

В этой же статье [8] Л. Гирайтис и Д. Сургайлис выдвинули гипотезу о том, что одного условия конечности интеграла в правой части (1.10) достаточно для выполнения (1.10). В работе [6] один из авторов опроверг эту гипотезу. Чтобы сформулировать этот результат, рассмотрим функции

И

$$
f_{0}(\lambda)=\left\{\begin{array}{lll}
\left(\frac{2^{s}}{s^{2}}\right)^{1 / p}, & \text { если } \quad 2^{-s-1} \leqslant \lambda \leqslant 2^{-s}, \quad s=2 m, \\
0, & \text { если } \quad 2^{-s-1} \leqslant \lambda \leqslant 2^{-s}, \quad s=2 m+1,
\end{array}\right.
$$

$$
g_{0}(\lambda)=\left\{\begin{array}{lll}
\left(\frac{2^{s}}{s^{2}}\right)^{1 / q}, & \text { если } \quad 2^{-s-1} \leqslant \lambda \leqslant 2^{-s}, \quad s=2 m+1, \\
0, & \text { если } 2^{-s-1} \leqslant \lambda \leqslant 2^{-s}, \quad s=2 m,
\end{array}\right.
$$

где $m-$ натуральное число и $p, q \geqslant 1$.

Легко видеть, что $f_{0}(\lambda) \in L^{p}(\mathbb{T}), g_{0}(\lambda) \in L^{q}(\mathbb{T}), f_{0}(\lambda) g_{0}(\lambda) \in L^{r}(\mathbb{T})$ для любого $r$ и

$$
\sigma^{2}=16 \pi^{3} \int_{-\pi}^{\pi} f_{0}^{2}(\lambda) g_{0}^{2}(\lambda) d \lambda=0 .
$$

С другой стороны, как было доказано в [6], при $\frac{1}{p}+\frac{1}{q}>1$

$$
\chi_{2}\left(\widetilde{Q}_{n}\right)=\frac{2}{n} \operatorname{tr}\left(T_{n}\left(f_{0}\right) T_{n}\left(g_{0}\right)\right)^{2} \longrightarrow \infty \quad \text { при } \quad n \rightarrow \infty,
$$

т.е. сходимость в (1.10) не имеет места.

В [6] было высказано предположение, что для сходимости в (1.10) достаточно условия

$$
0<\int_{-\pi}^{\pi} f^{2}(\lambda) g^{2}(\lambda) d \lambda<\infty
$$

Проблема b) - описание предельного распределения квадратичной формы $Q_{n}$, если оно негауссовское, была исследована Н. Террин и М. С. Такку в [14] и [15]. Пусть $f(\lambda)=|\lambda|^{-\alpha} L_{1}(\lambda)$ и $g(\lambda)=|\lambda|^{-\beta} L_{2}(\lambda)$, где $L_{1}(\lambda)$ и $L_{2}(\lambda)$ - медленно меняющиеся в точке $\lambda=0$ ограниченные функции. В [14] и [15] было доказано, что если $\alpha<1, \beta<1$ и $\alpha+\beta>\frac{1}{2}$, то случайная величина

$$
\widehat{Q}_{n}=\frac{1}{n^{\alpha+\beta} L_{1}(1 / n) L_{2}(1 / n)}\left(Q_{n}-\mathbf{E} Q_{n}\right)
$$


сходится по распределению к некоторой негауссовской случайной величине $Y(\alpha, \beta)$, которая может быть представлена посредством двойного интеграла Винера-Ито.

Отметим, что наличие слабо меняющихся функций $L_{1}(\lambda)$ и $L_{2}(\lambda)$ существенно, так как они дают бо́льшую свободу при выборе функций $f(\lambda)$ и $g(\lambda)$. В работе [14] было установлено, что эти функции влияют только на нормировку $Q_{n}$ (см. (1.15)), а предел $Y(\alpha, \beta)$ от них не зависит. Из результатов настоящей работы следует, что в критическом случае $\alpha+\beta=\frac{1}{2}$ предельное распределение стандартно нормированной квадратичной формы $Q_{n}$ уже зависит от функций $L_{1}(\lambda)$ и $L_{2}(\lambda)$.

Критический случай $\alpha+\beta=\frac{1}{2}$ был частично исследован Н. Террин и М.С. Такку в [16]. Исходя из величины $Y(\alpha, \beta)$, которая сушествует только при $\alpha+\beta>\frac{1}{2}$, они доказали, что если $0<\alpha<1,0<\beta<1$, то случайная величина $\left(\alpha+\beta-\frac{1}{2}\right) Y(\alpha, \beta)$ сходится по распределению к гауссовской случайной величине, когда $\alpha+\beta$ стремится к $\frac{1}{2}$.

В настоящей работе, предположив, что $f(\lambda)$ и $g(\lambda)$ - регулярно меняющиеся в точке $\lambda=0$ функции порядка $\alpha$ и $\beta$ соответственно, мы доказываем центральную предельную теорему (ЦПТ) для стандартно нормированной квадратичной формы $Q_{n}$ в критическом случае $\alpha+\beta=\frac{1}{2}$. Мы также показываем, что условие положительности и конечности асимптотической дисперсии квадратичной формы $Q_{n}$ не гарантирует выполнение ЦПТ для $Q_{n}$.

2. Основные результаты. Пусть $S V$ - класс медленно меняющихся в нуле функций $u(\lambda)$ с условиями: $u(\lambda) \in L^{\infty}(\mathbf{R}), \lim _{\lambda \rightarrow 0} u(\lambda)=0$, $u(\lambda)=u(-\lambda)$ и $0<u(\lambda)<u(\mu)$ при $0<\lambda<\mu$.

Теорема 2.1. Пусть

$$
\begin{aligned}
f(\lambda) & \leqslant|\lambda|^{-\alpha} L_{1}(\lambda), \\
|g(\lambda)| & \leqslant|\lambda|^{-\beta} L_{2}(\lambda),
\end{aligned}
$$

$2 \partial e$

$$
\begin{gathered}
\alpha<1, \quad \beta<1, \quad \alpha+\beta \leqslant \frac{1}{2}, \\
L_{i}(\lambda) \in S V, \quad \lambda^{-(\alpha+\beta)} L_{i}(\lambda) \in L^{2}(\mathbb{T}), \quad i=1,2 .
\end{gathered}
$$

Тогда имеет место (1.5), где дисперсия $\sigma^{2}$ определена в (1.7).

3 а м е ч а н и е 2.1. В качестве примера спектральной плотности $f(\lambda)$ и порождающей функции $g(\lambda)$, удовлетворяющих условиям теоремы 2.1, можно рассмотреть функции

$$
f(\lambda)=|\lambda|^{-\alpha}|\ln | \lambda||^{-\gamma} \quad \text { и } \quad g(\lambda)=|\lambda|^{-\beta}|\ln | \lambda||^{-\gamma}
$$

где $\alpha<1, \beta<1, \alpha+\beta \leqslant \frac{1}{2}$ и $\gamma>\frac{1}{2}$. 
3 а м е ч а н и е 2.2. В теореме 2.1 функции $f(\lambda)$ и $g(\lambda)$ имеют особенности в точке нуль и ограничены вне произвольной окрестности этой точки. Ясно, что выбор точки нуль здесь несущественен и вместо нее можно брать произвольную точку $\lambda_{0} \in[-\pi, \pi]$. Используя свойства произведения тёплицевых матриц $T_{n}(f)$ и $T_{n}(g)$, можно показать, что утверждение теоремы 2.1 остается в силе также в случае, когда $f(\lambda)$ и $g(\lambda)$ имеют особенности вида $(2.1),(2.2)$ в одном и том же конечном числе точек отрезка $[-\pi, \pi]$.

Для функций $f, g \in L^{1}(\mathbb{T})$ положим

$$
\varphi\left(t_{1}, t_{2}, t_{3}\right)=\int_{-\pi}^{\pi} f(u) g\left(u-t_{1}\right) f\left(u-t_{2}\right) g\left(u-t_{3}\right) d u .
$$

Теорема 2.2. Если функиия $\varphi\left(t_{1}, t_{2}, t_{3}\right) \in L^{2}\left(\mathbb{T}^{3}\right)$ непрерьвна в точке $(0,0,0)$, то имеет место (1.5) с дисперсией $\sigma^{2}$, определенной в (1.7).

Утверждение 2.1. Из теоремь 2.2 вытекают теоремы $\mathrm{A} u \mathrm{D}$.

3 а м е ч а н и е 2.3. Для функций $f(\lambda)=\lambda^{-3 / 4}$ и $g(\lambda)=\lambda^{3 / 4}$, удовлетворяющих условиям теоремы $\mathrm{B}$, функция $\varphi\left(t_{1}, t_{2}, t_{3}\right)$ не определена при $t_{2}=0, t_{1} \neq 0, t_{3} \neq 0$. Это значит, что, вообще говоря, из теоремы 2.2 не вытекает теорема В.

Следующее утверждение показывает, что положительность и конечность асимптотической дисперсии квадратичной формы $Q_{n}$ не гарантирует выполнение ЦПТ для $Q_{n}$.

Утверждение 2.2. Существуют спектральная плотность $f(\lambda)$ u порождающая функиия $g(\lambda)$ такие, что

$$
0<\int_{-\pi}^{\pi} f^{2}(\lambda) g^{2}(\lambda) d \lambda<\infty
$$

$u$

$$
\lim _{n \rightarrow \infty} \sup \chi_{2}\left(\widetilde{Q}_{n}\right)=\lim _{n \rightarrow \infty} \sup \frac{2}{n} \operatorname{tr}\left[T_{n}(f) T_{n}(g)\right]^{2}=\infty,
$$

т.е. условие (2.5) не гарантирует сходимость в (1.10).

3. Предварительные результаты. Напомним (см. [3], [13]), что положительная функция $u(x)$ называется медленно меняющейся в нуле, если $\lim _{x \rightarrow 0}[u(\lambda x) / u(x)]=1$ для любого $\lambda>0$. Отметим некоторые свойства медленно меняющихся функций, которые будут использованы ниже. Следующее свойство хорошо известно (см., например, [13]).

Лемма 3.1. Пусть $u(x)$ u $v(x), x \in \mathbb{R},-$ медленно меняюшиеся в нуле функции. Тогда 
a) для любого $p<1$

$$
\int_{0}^{y} x^{-p} u(x) d x=O\left(y^{1-p} u(y)\right) \quad \text { npu } \quad y \rightarrow 0 ;
$$

b) функиия $x^{p} u(x)$ возрастает на некотором интервале $(0, \delta)$, если $p>0$, и убьгвает, если $p<0$;

c) $u(x) v(x) u u(x) / v(x)$ - медленно меняюшиеся в нуле функиии.

Лемма 3.2. Для произвольных функиий $u, v \in S V$ и чисел $p, q<1$, $p+q>1$, существует постоянная $M>0$ такая, что

$$
\int_{\mathbb{T}}|x|^{-p}|x-y|^{-q} u(x) v^{-1}(x-y) d x \leqslant M|y|^{1-p-q} u(y) v^{-1}(y), \quad y \in \mathbb{T} \text {. (3.1) }
$$

Д о к а з а т е ль с т в о. Положим

$$
Q(x, y)=|x|^{-p}|x-y|^{-q} u(x) v^{-1}(x-y) .
$$

Легко видеть, что для любого $\delta>0$

$$
\sup _{|y|>\delta} \int_{\mathbb{T}} Q(x, y) d x<\infty \quad \text { и } \quad \min _{|y|>\delta} y^{1-p-q} u(y) v^{-1}(y)>0 .
$$

Следовательно, достаточно доказать (3.1) для $y \in(-\delta, \delta)$ при достаточно малом $\delta>0$. Применив лемму $3.1 \mathrm{a})$, получим, что

$$
\begin{aligned}
\int_{0<|x|<|y| / 2} Q(x, y) d x & \leqslant\left(\frac{|y|}{2}\right)^{-q} v^{-1}\left(\frac{y}{2}\right) \int_{0<|x|<|y| / 2}|x|^{-p} u(x) d x \\
& \leqslant C y^{1-p-q} u(y) v(y) \\
\int_{|y| / 2<|x|<2|y|} Q(x, y) d x & \leqslant\left(\frac{|y|}{2}\right)^{-p} u(2|y|) \int_{|y| / 2<|x|<2|y|}|x-y|^{-q} v^{-1}(x-y) d x \\
& \leqslant C|y|^{-p} u(|y|) \int_{0<|x|<4|y|}|x|^{-q} v^{-1}(x) d x \\
& \leqslant C y^{1-p-q} u(y) v(y) \\
\int_{2|y|<|x|<\pi} Q(x, y) d x & \leqslant|y|^{-p} v^{-1}(y) \int_{2|y|<|x|<\pi}|x|^{-q} u(x) d x \\
& \leqslant C y^{1-p-q} u(y) v(y)
\end{aligned}
$$

Из (3.2)-(3.4) получим (3.1). Лемма 3.2 доказана.

Следуюшая лемма доказывается аналогичным образом.

Лемма 3.3. Пусть Функиии $u, w \in S V$ таковьь, что

$$
\int_{\mathbb{T}} x^{-1} u(x) w^{-3}(x) d x<\infty
$$


Тогда для любого $q \in(0,1)$ существует постоянная $M>0$ такая, ито

$$
\int_{\mathbb{T}}|x|^{-1}|x-y|^{-q} u(x) w^{-2}(x) w^{-1}(x-y) d x \leqslant M|y|^{-q} w^{-3}(y), \quad y \in \mathbb{T} .
$$

Обозначим через $D_{n}(x)$ ядро Дирихле:

$$
D_{n}(x)=\frac{\sin (n x / 2)}{\sin (x / 2)}
$$

Легко видеть, что

$$
\left|D_{n}(x)\right| \leqslant \min \left\{n,|x|^{-1}\right\} \quad \text { и } \quad\left|D_{n}(x)\right| \leqslant C n \psi_{n}(x), \quad x \in \mathbb{T},
$$

где $\psi_{n}(x)=(1+n|x|)^{-1}$.

Лемма 3.4. Для произвольной функиии $w \in S V$ и любого иисла $t \in(0,1)$ существует постоянная $M>0$ такая, ито

$$
\left|D_{n}(x)\right| \leqslant M w\left(n^{-1}\right) n^{t}|x|^{t-1} w^{-1}(x) .
$$

Д о к а з а т ел ь с т в о. Из леммы 3.1 b) следует, что функции $x^{t-1} w^{-1}(x)$ и $x^{-t} w(x)$ убывают на некотором интервале $(0, \delta)$. Так как

$$
\min \left\{w\left(n^{-1}\right) n^{t}|x|^{t-1} w^{-1}(x)\right\}>0
$$

мы можем предположить, что $n^{-1}<\delta$ и $|x|<\delta$. Теперь если $|x| \leqslant n^{-1}$, то из условия $n^{1-t} w^{-1}\left(n^{-1}\right) \leqslant x^{t-1} w^{-1}(x)$ и (3.6) следует, что

$$
\left|D_{n}(x)\right| \leqslant n=w\left(n^{-1}\right) n^{t} n^{1-t} w^{-1}\left(n^{-1}\right) \leqslant w\left(n^{-1}\right) n^{t}|x|^{t-1} w^{-1}(x) .
$$

Случай $|x|>n^{-1}$ доказывается аналогично. Лемма 3.4 доказана.

Следующая лемма доказана в [8].

Лемма 3.5. Для любого $\delta \in(0,1)$ существует постоянная $C_{\delta}>0$ такая, ито

$$
n \int_{\mathbb{T}} \psi_{n}(x-y) \psi_{n}(x-z) d x \leqslant C_{\delta} \psi_{n}^{1-\delta}(y-z), \quad y, z \in \mathbb{T} .
$$

Обозначим

$$
\Phi_{n}\left(x_{1}, x_{2}, x_{3}\right)=\frac{1}{(2 \pi)^{3} n} D_{n}\left(x_{1}\right) D_{n}\left(x_{2}\right) D_{n}\left(x_{3}\right) D_{n}\left(x_{1}+x_{2}+x_{3}\right),
$$

где $D_{n}(x)$ определено в (3.5). Для заданного $\alpha \in(0, \pi)$ мы полагаем

$$
\begin{aligned}
& \mathbb{E}_{\alpha}=\{|\mathbf{x}| \leqslant \alpha\}=\left\{\left(x_{1}, x_{2}, x_{3}\right) ;\left|x_{k}\right| \leqslant \alpha, k=1,2,3\right\}, \\
& \mathbb{E}_{\alpha}^{c}=\{|\mathbf{x}| \leqslant \pi\} \backslash\{|\mathbf{x}| \leqslant \alpha\} .
\end{aligned}
$$


Лемма 3.6. Ядро $\Phi_{n}(\mathbf{x})$, определенное в (3.7), с $\mathbf{x}=\left(x_{1}, x_{2}, x_{3}\right)$ удовлетворяет следующим условиям:

a) $\int_{\mathbb{T}^{3}} \Phi_{n}(\mathbf{x}) d \mathbf{x}=1$

b) $\sup _{n} \int_{\mathbb{T}^{3}}\left|\Phi_{n}(\mathbf{x})\right| d \mathbf{x}=C_{1}<\infty$;

c) для любого $\varepsilon \in(0, \pi)$ имеем $\lim _{n \rightarrow \infty} \int_{\mathbb{E}_{\varepsilon}^{c}}\left|\Phi_{n}(\mathbf{x})\right| d \mathbf{x}=0$;

d) для любого $\delta>0$ существует постоянная $M_{\delta}>0$ такая, что

$$
\int_{\mathbb{E}_{\delta}^{c}} \Phi_{n}^{2}(\mathbf{x}) d \mathbf{x} \leqslant M_{\delta}, \quad n=1,2, \ldots .
$$

Д о к а з а т е л ь с т в о. Доказательство утверждений а)-с) можно найти в [2] (лемма 3.1). Для доказательства d) сперва заметим, что

$$
\int_{\mathbb{T}} D_{n}^{2}(x) d x \leqslant C n \quad \text { и } \quad\left|D_{n}(x)\right| \leqslant C_{\delta} \quad \text { при } \quad|x|>\delta, n=1,2, \ldots,
$$

где $D_{n}(x)$ - ядро Дирихле, а $C$ и $C_{\delta}$ - некоторые положительные константы. Имеем

$$
\begin{aligned}
\int_{\mathbb{E}_{\delta}^{c}} \Phi_{n}^{2}(\mathbf{x}) d \mathbf{x} & \leqslant \int_{\left|x_{1}\right|>\delta} \Phi_{n}^{2}(\mathbf{x}) d \mathbf{x}+\int_{\left|x_{2}\right|>\delta} \Phi_{n}^{2}(\mathbf{x}) d \mathbf{x}+\int_{\left|x_{3}\right|>\delta} \Phi_{n}^{2}(\mathbf{x}) d \mathbf{x} \\
& =: I_{1}+I_{2}+I_{3} .
\end{aligned}
$$

Очевидно, достаточно оценить $I_{1}$. Имеем

$$
\begin{aligned}
I_{1} \leqslant & \int_{\left|x_{1}\right|>\delta,\left|x_{2}\right|>\delta / 3} \Phi_{n}^{2}(\mathbf{x}) d \mathbf{x}+\int_{\left|x_{1}\right|>\delta,\left|x_{3}\right|>\delta / 3} \Phi_{n}^{2}(\mathbf{x}) d \mathbf{x} \\
& +\int_{\left|x_{1}\right|>\delta,\left|x_{2}\right| \leqslant \delta / 3,\left|x_{3}\right| \leqslant \delta / 3} \Phi_{n}^{2}(\mathbf{x}) d \mathbf{x}=: I_{1}^{(1)}+I_{1}^{(2)}+I_{1}^{(3)} .
\end{aligned}
$$

Используя (3.9), получим

$$
I_{1}^{(1)} \leqslant C_{\delta} \frac{1}{n^{2}} \int_{\mathbb{T}^{3}} D_{n}^{2}\left(x_{3}\right) D_{n}^{2}\left(x_{1}+x_{2}+x_{3}\right) d x_{1} d x_{2} d x_{3} \leqslant M_{\delta} .
$$

Аналогично,

$$
I_{1}^{(2)} \leqslant M_{\delta}
$$

Теперь, заметив, что в интеграле $I_{1}^{(3)}$ область интегрирования такова, что $\left|x_{1}+x_{2}+x_{3}\right|>\delta / 3$, из (3.9) находим

$$
I_{1}^{(3)} \leqslant C_{\delta} \frac{1}{n^{2}} \int_{\mathbb{T}^{3}} D_{n}^{2}\left(x_{2}\right) D_{n}^{2}\left(x_{3}\right) d x_{1} d x_{2} d x_{3} \leqslant M_{\delta} .
$$

Из (3.12)-(3.14) получаем (3.8). Лемма 3.6 доказана.

Лемма 3.7. Пусть Функция $\Psi(\mathbf{u}) \in L^{2}\left(\mathbb{T}^{3}\right)$ непрерьвна в точке $0=(0,0,0)$. Тогда

$$
\lim _{n \rightarrow \infty} \int_{\mathbb{T}^{3}} \Psi(\mathbf{u}) \Phi_{n}(\mathbf{u}) d \mathbf{u}=\Psi(\mathbf{0}),
$$

где $\mathbf{u}=\left(u_{1}, u_{2}, u_{3}\right)$, а функиия $\Phi_{n}(\mathbf{u})$ определена в (3.7). 
Д о к а з а т ел ь с т в. о. В силу леммы 3.6 a)

$$
R_{n}:=\int_{\mathbb{T}^{3}} \Psi(\mathbf{u}) \Phi_{n}(\mathbf{u}) d \mathbf{u}-\Psi(\mathbf{0})=\int_{\mathbb{T}^{3}}[\Psi(\mathbf{u})-\Psi(\mathbf{0})] \Phi_{n}(\mathbf{u}) d \mathbf{u} .
$$

Для любого $\varepsilon>0$ можно найти $\delta>0$ такое, что

$$
|\Psi(\mathbf{u})-\Psi(\mathbf{0})|<\frac{\varepsilon}{C_{1}}, \quad \text { если } \mathbf{u} \in \mathbb{E}_{\delta}
$$

где $C_{1}$ - постоянная из леммы $3.6 \mathrm{~b}$ ). Рассмотрим разложение $\Psi=$ $\Psi_{1}+\Psi_{2}$ такое, что

$$
\left\|\Psi_{1}\right\|_{2} \leqslant \frac{\varepsilon}{\sqrt{M_{\delta}}} \text { и }\left\|\Psi_{2}\right\|_{\infty}<\infty
$$

где $M_{\delta}$ - постоянная из леммы $3.6 \mathrm{~d}$ ). Применив лемму $\left.3.6 \mathrm{~b}\right)-\mathrm{d}$ ) и (3.16)-(3.18), находим, что при достаточно большом $n$

$$
\begin{aligned}
\left|R_{n}\right| \leqslant & \int_{\mathbb{E}_{\delta}}|\Psi(\mathbf{u})-\Psi(\mathbf{0})|\left|\Phi_{n}(\mathbf{u})\right| d \mathbf{u}+\int_{\mathbb{E}_{\delta}^{c}}\left|\Psi_{1}(\mathbf{u})\right|\left|\Phi_{n}(\mathbf{u})\right| d \mathbf{u} \\
& +\int_{\mathbb{E}_{\delta}^{c}}\left|\Psi_{2}(\mathbf{u})-\Psi(\mathbf{0})\right|\left|\Phi_{n}(\mathbf{u})\right| d \mathbf{u} \leqslant \frac{\varepsilon}{C_{1}} \int_{\mathbb{E}_{\delta}}\left|\Phi_{n}(\mathbf{u})\right| d \mathbf{u} \\
& +\left\|\Psi_{1}\right\|_{2}\left[\int_{\mathbb{E}_{\delta}^{c}} \Phi_{n}^{2}(\mathbf{u}) d \mathbf{u}\right]^{1 / 2}+C_{2} \int_{\mathbb{E}_{\delta}^{c}}\left|\Phi_{n}(\mathbf{u})\right| d \mathbf{u} \leqslant 3 \varepsilon .
\end{aligned}
$$

Отсюда с учетом (3.16) получаем (3.15). Лемма 3.7 доказана.

\section{4. Доказательства основных результатов.}

Док аз а тельст во т ео ремы 2.1. Для $f, g \in L^{1}(\mathbb{T})$ и $\mathbf{x}=\left(x_{1}, x_{2}, x_{3}, x_{4}\right)$ положим

$$
F(\mathbf{x})=f\left(x_{1}\right) f\left(x_{2}\right) g\left(x_{3}\right) g\left(x_{4}\right),
$$

и пусть

$$
H_{n}(\mathbf{x})=G_{n}\left(x_{1}-x_{3}\right) G_{n}\left(x_{2}-x_{3}\right) G_{n}\left(x_{4}-x_{1}\right) G_{n}\left(x_{4}-x_{2}\right),
$$

где

$$
G_{n}(u)=\sum_{k=1}^{n} e^{i k u}=e^{i u(n+1) / 2} D_{n}(u) .
$$

Легко проверить, что

$$
\operatorname{tr}\left(T_{n}(f) T_{n}(g)\right)^{2}=\int_{\mathbb{T}^{4}} F(\mathbf{x}) H_{n}(\mathbf{x}) d \mathbf{x} .
$$

Согласно теореме $\mathrm{B}$, достаточно рассмотреть случай $\alpha+\beta=\frac{1}{2}$. Тогда, в силу теоремы $\mathrm{C}$ нам нужно доказать, что

$$
\lim _{n \rightarrow \infty} \frac{1}{n} \int_{\mathbb{T}^{4}} F(\mathbf{x}) H_{n}(\mathbf{x}) d \mathbf{x}=8 \pi^{3} \int_{\mathbb{T}} f^{2}(x) g^{2}(x) d x
$$


при условии, что

$$
f(x) \leqslant|x|^{-\alpha} L(x), \quad|g(x)| \leqslant|x|^{-\beta} L(x), \quad x \in \mathbb{T},
$$

где $L=L_{1}+L_{2} \in S V$ и

$$
\alpha<1, \quad \beta<1, \quad \alpha+\beta=\frac{1}{2}, \quad \int_{\mathbb{T}} x^{-1} L^{2}(x) d x<\infty .
$$

Если $\alpha, \beta \geqslant 0$, то из (4.4) следует, что $f \in L^{1 / \alpha}(\mathbb{T}), g \in L^{1 / \beta}(\mathbb{T})$, и теорема 2.1 вытекает из теоремы А. Предположив, что $\beta<0$, из (4.5) имеем

$$
\frac{1}{2}<\alpha<1, \quad-\frac{1}{2}<\beta<0 .
$$

Для $\varepsilon \in(0,1)$ положим

$$
f_{\varepsilon}(x)=\left\{\begin{array}{lll}
0, & \text { если } & |x|<\varepsilon \\
f(x), & \text { если } & \varepsilon \leqslant|x| \leqslant \pi
\end{array}\right.
$$

и

$$
\mathbb{T}_{i, \varepsilon}=\left\{\mathbf{x} \in \mathbb{T}^{4}:\left|x_{i}\right|<\varepsilon\right\}, \quad i=1,2
$$

Имеем

$$
\frac{1}{n} \int_{\mathbb{T}^{4}} F(\mathbf{x}) H_{n}(\mathbf{x}) d \mathbf{x}=J_{n}^{1}+J_{n}^{2}
$$

где

$$
J_{n}^{1}:=\frac{1}{n} \int_{\mathbb{T}^{4}} f_{\varepsilon}\left(x_{1}\right) f_{\varepsilon}\left(x_{2}\right) g\left(x_{3}\right) g\left(x_{4}\right) H_{n}(\mathbf{x}) d \mathbf{x}
$$

и

$$
\left|J_{n}^{2}\right| \leqslant \frac{1}{n} \int_{\mathbb{T}_{1, \varepsilon}}\left|F(\mathbf{x}) H_{n}(\mathbf{x})\right| d \mathbf{x}+\frac{1}{n} \int_{\mathbb{T}_{2, \varepsilon}}\left|F(\mathbf{x}) H_{n}(\mathbf{x})\right| d \mathbf{x}=: I_{n}^{1}+I_{n}^{2} .
$$

Taк как $f_{\varepsilon}, g \in L^{\infty}(\mathbb{T})$, то

$$
\lim _{n \rightarrow \infty} J_{n}^{1}=8 \pi^{3} \int_{\mathbb{T}} f_{\varepsilon}^{2}(x) g^{2}(x) d x .
$$

Последний интеграл стремится к $\int_{\mathbb{T}} f^{2}(x) g^{2}(x) d x$, когда $\varepsilon \rightarrow 0$, значит, (4.3) будет следовать из того, что

$$
\lim _{\varepsilon \rightarrow 0, n \rightarrow \infty}\left(I_{n}^{1}+I_{n}^{2}\right)=0 .
$$

Достаточно доказать (4.7) для $I_{n}^{1}$. Положим

$$
\begin{aligned}
B_{i, j} & =\left\{\mathrm{x} \in \mathbb{T}^{4}:\left|x_{i}\right| \leqslant \frac{\left|x_{j}\right|}{2}\right\}, \quad i=1,2, \quad j=3,4, \\
B & =\left\{\mathrm{x} \in \mathbb{T}^{4}:\left|x_{1}\right|<\varepsilon,\left|x_{i}\right|>\frac{\left|x_{j}\right|}{2}, i=1,2, j=3,4\right\} .
\end{aligned}
$$


Тогда

$$
I_{n}^{1} \leqslant \frac{1}{n} \sum_{i=1}^{2} \sum_{j=3}^{4} \int_{B_{i, j}} F(\mathbf{x}) H_{n}(\mathbf{x}) d \mathbf{x}+\frac{1}{n} \int_{B} F(\mathbf{x}) H_{n}(\mathbf{x}) d \mathbf{x} .
$$

Допустим, что функция $w \in S V$ удовлетворяет условию

$$
\int_{\mathbb{T}} x^{-1} L^{2}(x) w^{-4}(x) d x<\infty .
$$

Так как

$$
\frac{\left|x_{3}\right|}{2}<\left|x_{1}-x_{3}\right|<2\left|x_{3}\right| \quad \text { при } \mathbf{x} \in B_{1,3},
$$

из оценок (4.4) и из леммы 3.4 следует, что

$$
\begin{aligned}
A_{1,3}:= & \frac{1}{n} \int_{B_{1,3}} F(\mathbf{x}) G_{n}(\mathbf{x}) d \mathbf{x} \\
\leqslant & C w^{4}\left(\frac{1}{n}\right) \int_{B_{1,3}}\left|x_{1}\right|^{-\alpha}\left|x_{2}\right|^{-\alpha}\left|x_{3}\right|^{-\beta}\left|x_{4}\right|^{-\beta} L\left(x_{1}\right) L\left(x_{2}\right) L\left(x_{3}\right) L\left(x_{4}\right) \\
& \times\left|x_{1}-x_{3}\right|^{-3 / 4}\left|x_{2}-x_{3}\right|^{-3 / 4}\left|x_{1}-x_{4}\right|^{-3 / 4}\left|x_{2}-x_{4}\right|^{-3 / 4} \\
& \times w^{-1}\left(x_{1}-x_{3}\right) w^{-1}\left(x_{2}-x_{3}\right) w^{-1}\left(x_{1}-x_{4}\right) w^{-1}\left(x_{2}-x_{4}\right) d \mathbf{x} \\
\leqslant & C w^{4}\left(\frac{1}{n}\right) \int_{\mathbb{T}^{2}}\left|x_{2}\right|^{-\alpha}\left|x_{4}\right|^{-\beta}\left|x_{2}-x_{4}\right|^{-3 / 4} L\left(x_{2}\right) L\left(x_{4}\right) w^{-1}\left(x_{2}-x_{4}\right) \\
& \times \int_{\mathbb{T}}\left|x_{1}\right|^{-\alpha}\left|x_{1}-x_{4}\right|^{-3 / 4} L\left(x_{1}\right) w^{-1}\left(x_{1}-x_{4}\right) d x_{1} \\
& \times \int_{\mathbb{T}}\left|x_{3}\right|^{-\beta-3 / 4}\left|x_{2}-x_{3}\right|^{-3 / 4} L\left(x_{3}\right) w^{-1}\left(x_{3}\right) w^{-1}\left(x_{2}-x_{3}\right) d x_{3} d x_{2} d x_{4} .
\end{aligned}
$$

Применив сначала лемму 3.2 , затем лемму 3.3 , находим, что

$$
\begin{aligned}
A_{1,3} \leqslant & C w^{4}\left(\frac{1}{n}\right) \int_{\mathbb{T}^{2}}\left|x_{2}\right|^{-\alpha}\left|x_{4}\right|^{-\beta}\left|x_{2}-x_{4}\right|^{-3 / 4} L\left(x_{2}\right) L\left(x_{4}\right) w^{-1}\left(x_{2}-x_{4}\right) \\
& \times\left|x_{4}\right|^{-\alpha+1 / 4} L\left(x_{4}\right) w^{-1}\left(x_{4}\right)\left|x_{2}\right|^{-\beta-1 / 2} L\left(x_{2}\right) w^{-2}\left(x_{2}\right) d x_{2} d x_{4} \\
= & C w^{4}\left(\frac{1}{n}\right) \int_{\mathbb{T}}\left|x_{4}\right|^{-1 / 4} L^{2}\left(x_{4}\right) w^{-1}\left(x_{4}\right) \\
& \times \int_{\mathbb{T}}\left|x_{2}\right|^{-1}\left|x_{2}-x_{4}\right|^{-3 / 4} L^{2}\left(x_{2}\right) w^{-2}\left(x_{2}\right) w^{-1}\left(x_{2}-x_{4}\right) d x_{2} d x_{4} \\
\leqslant & C w^{4}\left(\frac{1}{n}\right) \int_{\mathbb{T}}\left|x_{4}\right|^{-1} L^{2}\left(x_{4}\right) w^{-4}\left(x_{4}\right) d x_{4}=o(1),
\end{aligned}
$$

когда $n \rightarrow \infty$. Аналогичным образом можно доказать, что все интегралы под знаком суммы в (4.8) стремятся к нулю при $n \rightarrow \infty$. Для 
оценки последнего интеграла в (4.8) воспользуемся (4.4) и леммой 3.5:

$$
\begin{aligned}
& A:=\frac{1}{n} \int_{B}\left|F(\mathbf{x}) H_{n}(\mathbf{x})\right| d \mathbf{x} \\
& \leqslant C n^{3} \int_{B}\left|x_{1}\right|^{-\alpha}\left|x_{2}\right|^{-\alpha}\left|x_{3}\right|^{-\beta}\left|x_{4}\right|^{-\beta} L\left(x_{1}\right) L\left(x_{2}\right) L\left(x_{3}\right) L\left(x_{4}\right) \\
& \times \psi_{n}\left(x_{1}-x_{3}\right) \psi_{n}\left(x_{2}-x_{3}\right) \psi_{n}\left(x_{1}-x_{4}\right) \psi_{n}\left(x_{2}-x_{4}\right) d \mathbf{x} \\
& \leqslant C n^{3} \int_{(-2 \varepsilon, 2 \varepsilon)^{2}}\left|x_{3}\right|^{-1 / 2}\left|x_{4}\right|^{-1 / 2} L\left(x_{3}\right) L\left(x_{4}\right) \\
& \times \int_{\mathbb{T}} \psi_{n}\left(x_{1}-x_{3}\right) \psi_{n}\left(x_{1}-x_{4}\right) L\left(x_{1}\right) d x_{1} \\
& \times \int_{\mathbb{T}} \psi_{n}\left(x_{2}-x_{3}\right) \psi_{n}\left(x_{2}-x_{4}\right) L\left(x_{2}\right) d x_{2} d x_{3} d x_{4} \\
& \leqslant C n \int_{(-2 \varepsilon, 2 \varepsilon)}\left|x_{3}\right|^{-1 / 2} L\left(x_{3}\right) \int_{\mathbb{T}}\left|x_{4}\right|^{-1 / 2} \psi_{n}^{3 / 2}\left(x_{3}-x_{4}\right) L\left(x_{4}\right) d x_{4} d x_{3} \\
& \leqslant C \int_{-2 n \varepsilon}^{2 n \varepsilon}|y|^{-1 / 2} L\left(\frac{y}{n}\right) \int_{-\infty}^{\infty} \frac{|x|^{-1 / 2}}{(1+|x-y|)^{3 / 2}} L\left(\frac{x}{n}\right) d x d y .
\end{aligned}
$$

Докажем, что

$$
\int_{-\infty}^{\infty} \frac{|x|^{-1 / 2}}{(1+|x-y|)^{3 / 2}} L\left(\frac{x}{n}\right) d x \leqslant C y^{-1 / 2} L\left(\frac{y}{n}\right), \quad y \in \mathbb{T} .
$$

Действительно, при $y \in \mathbb{T}$

$$
\begin{aligned}
\int_{|x| \leqslant|y|} \frac{|x|^{-1 / 2}}{(1+|x-y|)^{3 / 2}} L\left(\frac{x}{n}\right) d x & \leqslant C L\left(\frac{y}{n}\right) \int_{\mathbb{T}}|x|^{-1 / 2} d x \\
& \leqslant C L\left(\frac{y}{n}\right) \leqslant C y^{-1 / 2} L\left(\frac{y}{n}\right) .
\end{aligned}
$$

Согласно лемме 3.1 , функция $t^{-1 / 2} L(t)$ убывает на некотором интервале $(0, \delta)$. Следовательно, предположив без ограничения общности, что $n>\pi / \delta$, для $|x|>|y|$ будем иметь

$$
\begin{aligned}
|x|^{-1 / 2} L\left(\frac{x}{n}\right) & =n^{-1 / 2}\left(\frac{|x|}{n}\right)^{-1 / 2} L\left(\frac{x}{n}\right) \leqslant n^{-1 / 2}\left(\frac{|y|}{n}\right)^{-1 / 2} L\left(\frac{y}{n}\right) \\
& =|y|^{-1 / 2} L\left(\frac{y}{n}\right) .
\end{aligned}
$$

Поэтому

$$
\begin{aligned}
\int_{|x|>|y|} \frac{|x|^{-1 / 2}}{(1+|x-y|)^{3 / 2}} L\left(\frac{x}{n}\right) d x & \leqslant C|y|^{-1 / 2} L\left(\frac{y}{n}\right) \int_{-\infty}^{\infty} \frac{1}{(1+|x|)^{3 / 2}} d x \\
& \leqslant C|y|^{-1 / 2} L\left(\frac{y}{n}\right) .
\end{aligned}
$$


Из (4.13), (4.14) мы получим (4.12), а из (4.11), (4.12) и (4.5) находим, что

$$
A \leqslant C \int_{-2 n \varepsilon}^{2 n \varepsilon}|y|^{-1} L^{2}\left(\frac{y}{n}\right) d y=C \int_{-2 \varepsilon}^{2 \varepsilon}|t|^{-1} L^{2}(t) d t=o(\varepsilon),
$$

когда $\varepsilon \rightarrow 0$. Из (4.8), (4.10) и (4.15) вытекает (4.7).

Теорема 2.1 доказана.

Д о к а зате л ь с т в о т е о р е мы 2.2. С помощью замены переменных $x_{1}=u, x_{1}-x_{3}=u_{1}, x_{3}-x_{2}=u_{2}$ и $x_{2}-x_{4}=u_{3}$ из $(4.2)$ получим:

$$
\begin{aligned}
\operatorname{tr}\left(T_{n}(f) T_{n}(g)\right)^{2}=\int_{\mathbb{T}^{4}} & G_{n}\left(u_{1}\right) G_{n}\left(u_{2}\right) G_{n}\left(u_{3}\right) G_{n}\left(-u_{1}-u_{2}-u_{3}\right) \\
\times & f(u) g\left(u-u_{1}\right) f\left(u-u_{1}-u_{2}\right) \\
& \times g\left(u-u_{1}-u_{2}-u_{3}\right) d u_{1} d u_{2} d u_{3} d u_{4},
\end{aligned}
$$

где $G_{n}(u)$ определено в (4.1). Учитывая равенство

$$
e^{i u_{1}(n+1) / 2} e^{i u_{2}(n+1) / 2} e^{i u_{3}(n+1) / 2} e^{-i\left(u_{1}+u_{2}+u_{3}\right)(n+1) / 2}=1
$$

и четность функции $D_{n}(u)$, из (4.16) находим, что

$$
\operatorname{tr}\left(T_{n}(f) T_{n}(g)\right)^{2}=8 \pi^{3} \int_{\mathbb{T}^{3}} \Psi\left(u_{1}, u_{2}, u_{3}\right) \Phi_{n}\left(u_{1}, u_{2}, u_{3}\right) d u_{1} d u_{2} d u_{3},
$$

где $\Phi_{n}\left(u_{1}, u_{2}, u_{3}\right)$ определено в $(3.7), \Psi\left(u_{1}, u_{2}, u_{3}\right)=\varphi\left(u_{1}, u_{1}+u_{2}, u_{1}+\right.$ $\left.u_{2}+u_{3}\right)$, а $\varphi\left(u_{1}, u_{2}, u_{3}\right)$ определено в (2.4). Согласно теореме С и (4.17), нам нужно доказать, что

$$
\lim _{n \rightarrow \infty} \int_{\mathbb{T}^{3}} \Psi(\mathbf{u}) \Phi_{n}(\mathbf{u}) d \mathbf{u}=\int_{\mathbb{T}} f^{2}(x) g^{2}(x) d x .
$$

Учитывая, что функции $\varphi\left(u_{1}, u_{2}, u_{3}\right)$ и $\Psi\left(u_{1}, u_{2}, u_{3}\right)$ интегрируемы с квадратом и непрерывны в точке $(0,0,0)$ одновременно, а

$$
\Psi(0,0,0)=\int_{\mathbb{T}} f^{2}(x) g^{2}(x) d x
$$

из леммы 3.7 получаем (4.18). Теорема 2.2 доказана.

Д оказатель с т в о у т в е р д ен и я 2.1. Для того чтобы доказать, что из теоремы 2.2 следует теорема $\mathrm{A}$, достаточно убедиться, что функция

$$
\varphi(\mathbf{t}):=\int_{\mathbb{T}} f_{0}(u) f_{1}\left(u-t_{1}\right) f_{2}\left(u-t_{2}\right) f_{3}\left(u-t_{3}\right) d u, \quad \mathbf{t}=\left(t_{1}, t_{2}, t_{3}\right),
$$

принадлежит $L^{2}\left(\mathbb{T}^{3}\right)$ и непрерывна в точке $(0,0,0)$ при условии, что

$$
f_{i} \in L^{p_{i}}(\mathbb{T}), \quad 1 \leqslant p_{i} \leqslant \infty, \quad i=0,1,2,3, \quad \sum_{i=0}^{3} \frac{1}{p_{i}} \leqslant 1 .
$$


Из неравенства Гёльдера и (4.20) следует, что

$$
|\varphi(\mathbf{t})| \leqslant \prod_{i=0}^{3}\left\|f_{i}\right\|_{L^{p_{i}}(\mathbb{T})}, \quad \mathbf{t}=\left(t_{1}, t_{2}, t_{3}\right) \in \mathbb{T}^{3}
$$

Следовательно, $\varphi(\mathbf{t}) \in L^{2}\left(\mathbb{T}^{3}\right)$. Для доказательства непрерывности $\varphi(\mathbf{t})$ в точке $(0,0,0)$ рассмотрим три случая.

Случай 1: $p_{i}<\infty, i=0,1,2,3$. Для произвольного $\varepsilon>0$ найдем $\delta>0$ с условием (см. (4.20))

$$
\left\|f_{i}(u-t)-f_{i}(u)\right\|_{p_{i}} \leqslant \varepsilon, \quad i=1,2,3, \text { если }|t| \leqslant \delta .
$$

Зафиксируем $\mathbf{t}=\left(t_{1}, t_{2}, t_{3}\right)$ с $|\mathbf{t}|<\delta$ и положим $\bar{f}_{i}(u)=f_{i}\left(u+t_{i}\right)-$ $f_{i}(u), i=1,2,3$. Тогда из (4.21) находим, что $\left\|\bar{f}_{i}\right\|_{p_{i}} \leqslant \varepsilon, i=1,2,3$, и, следовательно,

$$
\varphi(\mathbf{t})=\int_{\mathbb{T}} f_{0}(u) \prod_{i=1}^{3}\left(\bar{f}_{i}(u)+f_{i}(u)\right) d u=\varphi(0,0,0)+W,
$$

где величина $W$ есть сумма пяти интегралов. Каждый из них содержит по крайней мере одну функцию $\bar{f}_{i}$ и может быть оценен, как следующий интеграл:

$$
\left|\int_{\mathbb{T}} f_{0}(u) \bar{f}_{1}(u) f_{2}(u) f_{3}(u) d u\right| \leqslant\left\|f_{0}\right\|_{p_{0}}\left\|\bar{f}_{1}\right\|_{p_{1}}\left\|f_{2}\right\|_{p_{2}}\left\|f_{3}\right\|_{p_{3}} \leqslant C \varepsilon .
$$

Случай 2: $p_{i} \leqslant \infty, i=0,1,2,3, \sum_{i=0}^{3} 1 / p_{i}<1$. Существуют конечные числа $p_{i}^{\prime}<p_{i}, i=0,1,2,3, \sum_{i=0}^{3} 1 / p_{i}^{\prime} \leqslant 1$, для которых $f_{i} \in L^{p_{i}}(\mathbb{T})$. Следовательно, функция $\varphi$ непрерывна в $(0,0,0)$, как это доказывалось в случае 1.

Cлучай 3: $p_{i} \leqslant \infty, i=0,1,2,3, \sum_{i=0}^{3} 1 / p_{i}=1$.

По крайней мере одно из чисел $p_{i}$ конечно. Допустим, что $p_{0}<\infty$. Для любого $\varepsilon>0$ найдем функции $f_{0}^{\prime}$ и $f_{0}^{\prime \prime}$ такие, что

$$
f_{0}=f_{0}^{\prime}+f_{0}^{\prime \prime}, \quad f_{0}^{\prime} \in L^{\infty}, \quad\left\|f_{0}^{\prime \prime}\right\|_{p_{0}}<\varepsilon .
$$

Тогда

$$
\varphi(\mathbf{t})=\varphi^{\prime}(\mathbf{t})+\varphi^{\prime \prime}(\mathbf{t})
$$

где функции $\varphi^{\prime}$ и $\varphi^{\prime \prime}$ определяются так же, как функция $\varphi$ в (4.19), с заменой $f_{0}$ на $f_{0}^{\prime}$ и $f_{0}^{\prime \prime}$ соответственно. Из (4.22) следует, что $\varphi^{\prime}$ непрерывна в $(0,0,0)$ (см. случай 2$)$, а для $\varphi^{\prime \prime}$, применив неравенство Гёльдера, получим, что $\left|\varphi^{\prime \prime}(\mathbf{t})\right| \leqslant C \varepsilon$. Следовательно, для достаточно малых $|\mathbf{t}|$

$$
|\varphi(\mathbf{t})-\varphi(0,0,0)| \leqslant\left|\varphi^{\prime}(\mathbf{t})-\varphi^{\prime}(0,0,0)\right|+\left|\varphi^{\prime \prime}(\mathbf{t})-\varphi^{\prime \prime}(0,0,0)\right| \leqslant(C+1) \varepsilon
$$

откуда вытекает требуемый результат. 
Теперь докажем, что из теоремы 2.2 вытекает теорема D. Для этого достаточно показать, что функция

$$
\varphi(\mathbf{t})=\int_{\mathbb{T}} f(u) g\left(u-t_{1}\right) f\left(u-t_{2}\right) g\left(u-t_{3}\right) d u, \quad \mathbf{t}=\left(t_{1}, t_{2}, t_{3}\right) \in \mathbb{T}^{3},
$$

принадлежит $L^{2}\left(\mathbb{T}^{3}\right)$ и непрерывна в точке $(0,0,0)$, если $f$ и $g$ удовлетворяют условиям теоремы $\mathrm{D}$, т.е. $f \in L_{2}(\mathbb{T}), g \in L_{2}(\mathbb{T}), f g \in L_{2}(\mathbb{T})$ и имеет место (1.11).

Так как

$$
\varphi^{2}(\mathbf{t}) \leqslant 2 \pi \int_{\mathbb{T}} f^{2}(u) g^{2}\left(u-t_{1}\right) f^{2}\left(u-t_{2}\right) g^{2}\left(u-t_{3}\right) d u,
$$

то имеем

$$
\begin{aligned}
\int_{\mathbb{T}^{3}} \varphi^{2}(\mathbf{t}) d \mathbf{t} & \leqslant \int_{\mathbb{T}}\left[\int_{\mathbb{T}} g^{2}\left(u-t_{1}\right) d t_{1} \int_{\mathbb{T}} f^{2}\left(u-t_{2}\right) d t_{2} \int_{\mathbb{T}} g^{2}\left(u-t_{3}\right) d t_{3}\right] f^{2}(u) d u \\
& =\|f\|_{2}^{4}\|g\|_{2}^{4}<\infty .
\end{aligned}
$$

Теперь докажем непрерывность $\varphi(\mathbf{t})$ в точке $(0,0,0)$. Пусть $\varepsilon-$ произвольное положительное число. Положим

$$
E_{K}=\{u \in \mathbb{T}:|f(u)| \leqslant K\}, \quad f_{1}(u)=\chi_{E_{K}}(u) f(u), \quad f_{2}(u)=f(u)-f_{1}(u),
$$

где число $K>0$ выбрано так, чтобы выполнялось равенство

$$
\int_{\mathbb{T} \backslash E_{k}} f^{2}(u) g^{2}(u) d u \leqslant \varepsilon
$$

Тогда

$$
f=f_{1}+f_{2}, \quad\left\|f_{1}\right\|_{\infty} \leqslant K, \quad \int_{\mathbb{T}} f_{2}^{2}(u) g^{2}(u) d u \leqslant \varepsilon .
$$

Рассмотрим разложение

$$
\begin{aligned}
\varphi(\mathbf{t})= & \int_{\mathbb{T}} f_{1}(u) g\left(u-t_{1}\right) f_{1}\left(u-t_{2}\right) g\left(u-t_{3}\right) d u \\
& +\int_{\mathbb{T}} f_{2}(u) g\left(u-t_{1}\right) f\left(u-t_{2}\right) g\left(u-t_{3}\right) d u \\
& +\int_{\mathbb{T}} f_{1}(u) g\left(u-t_{1}\right) f_{2}\left(u-t_{2}\right) g\left(u-t_{3}\right) d u \\
=: & \varphi_{1}(\mathbf{t})+\varphi_{2}(\mathbf{t})+\varphi_{3}(\mathbf{t}) .
\end{aligned}
$$

Оценим функции $\varphi_{k}(\mathbf{t}), k=1,2,3$. Имеем

$$
\begin{aligned}
\varphi_{1}(\mathbf{t})= & \int_{\mathbb{T}} f_{1}(u) g\left(u-t_{1}\right) f_{1}\left(u-t_{2}\right)\left[g\left(u-t_{3}\right)-g(u)\right] d u \\
& +\int_{\mathbb{T}} f_{1}(u) g(u) f_{1}\left(u-t_{2}\right)\left[g\left(u-t_{1}\right)-g(u)\right] d u \\
& +\int_{\mathbb{T}} f_{1}(u) g^{2}(u) f_{1}\left(u-t_{2}\right) d u=: I_{1}+I_{2}+I_{3} .
\end{aligned}
$$


Применив неравенство Гёльдера, из (4.24) получим, что

$$
\left|I_{1}\right| \leqslant K^{2}\|g\|_{2}\left\|g\left(u+t_{3}\right)-g(u)\right\|_{2}=o(1) \quad \text { при } \quad t_{3} \rightarrow 0 .
$$

Аналогично,

$$
\left|I_{2}\right|=o(1) \quad \text { при } \quad t_{1} \rightarrow 0
$$

и, с учетом (4.24),

$$
\begin{aligned}
& \left|I_{3}-\int_{\mathbb{T}} \varphi(0,0,0)\right|=\left|\int_{\mathbb{T}} f_{1}\left(u+t_{2}\right) g^{2}\left(u+t_{2}\right) f_{1}(u) d u-\int_{\mathbb{T}} f_{1}^{2}(u) g^{2}(u) d u\right| \\
& \quad+\left|\int_{\mathbb{T}} f_{2}^{2}(u) g^{2}(u) d u\right| \\
& \leqslant K\left\|f_{1}\left(u+t_{2}\right) g^{2}\left(u+t_{2}\right)-f_{1}(u) g_{1}^{2}(u)\right\|_{1}+\varepsilon=o(1)+\varepsilon
\end{aligned}
$$

при $t_{2} \rightarrow 0$. Из (4.26)-(4.29) для достаточно малого $|\mathbf{t}|$ имеем

$$
\left|\varphi_{1}(\mathbf{t})-\varphi(0,0,0)\right| \leqslant 2 \varepsilon
$$

Далее, для $\varphi_{2}(\mathbf{t})$ имеем

$$
\begin{aligned}
\left|\varphi_{2}(\mathbf{t})\right|^{2} \leqslant & \int_{\mathbb{T}} f_{2}^{2}(u) g^{2}\left(u-t_{1}\right) d u \int_{\mathbb{T}} f_{2}^{2}\left(u-t_{2}\right) g^{2}\left(u-t_{3}\right) d u \\
= & \left|\int_{\mathbb{T}} f^{2}(u) g^{2}\left(u-t_{1}\right) d u-\int_{\mathbb{T}} f_{1}^{2}(u) g^{2}\left(u-t_{1}\right) d u\right| \\
& \times \int_{\mathbb{T}} f^{2}(u) g^{2}\left(u+t_{2}-t_{3}\right) d u \\
\longrightarrow & \left|\int_{\mathbb{T}} f^{2}(u) g^{2}(u) d u-\int_{\mathbb{T}} f_{1}^{2}(u) g^{2}(u) d u\right| \int_{\mathbb{T}} f^{2}(u) g^{2}(u) d u
\end{aligned}
$$

когда $|\mathbf{t}| \rightarrow 0$. Следовательно, с учетом (4.24), при достаточно малом $|\mathbf{t}|$

$$
\left|\varphi_{2}(\mathbf{t})\right| \leqslant \varepsilon \int_{\mathbb{T}} f^{2}(u) g^{2}(u) d u .
$$

Подобным образом можно доказать, что при достаточно малом $|\mathbf{t}|$

$$
\left|\varphi_{3}(\mathbf{t})\right| \leqslant \varepsilon \int_{\mathbb{T}} f^{2}(u) g^{2}(u) d u
$$

Из (4.25) и (4.30)-(4.32) вытекает, что

$$
\lim _{\mathbf{t} \rightarrow 0} \varphi(\mathbf{t})=\varphi(0,0,0) .
$$

Утверждение 2.1 доказано.

Доказательств о у в ерждения 2.2. Мы построим функции $f(\lambda)$ и $g(\lambda)$, удовлетворяющие условиям (2.5) и (2.6). Фиксировав $p \geqslant 2$, выберем число $q>1$ с условием $1 / p+1 / q>1$. Для этих $p$ 
и $q$ рассмотрим функции $f_{0}(\lambda)$ и $g_{0}(\lambda)$, определенные в $(1.12)$ и $(1.13)$ соответственно. Для произвольной положительной постоянной $C$ положим $g_{ \pm}(\lambda)=g_{0}(\lambda) \pm C$. Так как носители функций $f_{0}(\lambda)$ и $g_{0}(\lambda)$ не пересекаются, то

$$
\int_{-\pi}^{\pi} f_{0}^{2}(\lambda) g_{ \pm}^{2}(\lambda) d \lambda=\int_{-\pi}^{\pi} f_{0}^{2}(\lambda)\left(g_{0}(\lambda) \pm C\right)^{2} d \lambda=C^{2} \int_{-\pi}^{\pi} f_{0}^{2}(\lambda) d \lambda<\infty
$$

следовательно, условие (2.5) имеет место. Далее, в силу (1.14)

$$
\frac{1}{n} \operatorname{tr}\left(T_{n}\left(f_{0}\right) T_{n}\left(g_{0}\right)\right)^{2} \longrightarrow \infty \quad \text { при } \quad n \rightarrow \infty
$$

и в силу теоремы $\mathrm{B}$ с $p_{1}=p \geqslant 2$ и $p_{2}=\infty$

$$
\frac{1}{n} C^{2} \operatorname{tr}\left(T_{n}^{2}\left(f_{0}\right)\right) \longrightarrow 8 \pi^{3} C^{2} \int_{-\pi}^{\pi} f_{0}^{2}(\lambda) d \lambda<\infty .
$$

С другой стороны,

$$
\begin{aligned}
& \operatorname{tr}\left(T_{n}\left(f_{0}\right) T_{n}\left(g_{ \pm}\right)\right)^{2}=\operatorname{tr}\left(T_{n}\left(f_{0}\right) T_{n}\left(g_{0} \pm C\right)\right)^{2} \\
& \quad=\operatorname{tr}\left(T_{n}\left(f_{0}\right) T_{n}\left(g_{0}\right)\right)^{2} \pm 2 C \operatorname{tr}\left(T_{n}^{2}\left(f_{0}\right) T_{n}\left(g_{0}\right)\right)+C^{2} \operatorname{tr}\left(T_{n}^{2}\left(f_{0}\right)\right),
\end{aligned}
$$

откуда, с учетом (4.33) и (4.34), получим

$$
\begin{aligned}
& \frac{1}{n} \operatorname{tr}\left(T_{n}\left(f_{0}\right) T_{n}\left(g_{+}\right)\right)^{2}+\frac{1}{n} \operatorname{tr}\left(T_{n}\left(f_{0}\right) T_{n}\left(g_{-}\right)\right)^{2} \\
& \quad=\frac{2}{n} \operatorname{tr}\left(T_{n}\left(f_{0}\right) T_{n}\left(g_{0}\right)\right)^{2}+\frac{2}{n} C^{2} \operatorname{tr}\left(T_{n}^{2}\left(f_{0}\right)\right) \longrightarrow \infty \quad \text { при } \quad n \rightarrow \infty .
\end{aligned}
$$

Следовательно, либо

$$
\lim _{n \rightarrow \infty} \sup \frac{1}{n} \operatorname{tr}\left(T_{n}\left(f_{0}\right) T_{n}\left(g_{+}\right)\right)^{2}=\infty
$$

либо

$$
\lim _{n \rightarrow \infty} \sup \frac{1}{n} \operatorname{tr}\left(T_{n}\left(f_{0}\right) T_{n}\left(g_{-}\right)\right)^{2}=\infty .
$$

Таким образом,

$$
\lim _{n \rightarrow \infty} \sup \chi_{2}\left(\tilde{Q}_{n}\right)=\lim _{n \rightarrow \infty} \sup \frac{2}{n} \operatorname{tr}\left(T_{n}(f) T_{n}(g)\right)^{2}=\infty
$$

с $f=f_{0}$ и $g=g_{+}$или $g=g_{-}$. Утверждение 2.2 доказано.

Авторы выражают благодарность академику И. А. Ибрагимову за внимание к работе, а также рецензенту за полезные замечания и комментарии. 


\section{СПИСОК ЛИТЕРАТУРЫ}

1. Avram F. On bilinear forms in Gaussian random variables and Toeplitz matrices. Probab. Theory Related Fields, 1988, v. 79, № 1, p. 37-45.

2. Бенткус $P$. Об ошибке оценки спектральной функции стационарного процесса. Литов. матем. сб., 1972, т. 12, № 1, с. 55-71.

3. Феллер В. Введение в теорию вероятностей и ее приложения. Т. 2. М.: Мир, 1984, $752 \mathrm{c}$.

4. Fox R., Taqqu M.S. Central limit theorem for quadratic forms in random variables having long-range dependence. - Probab. Theory Related Fields, 1987, v. 74, № 2, p. 213-240.

5. Гиновян M. С. Асимптотически эффективное непараметрическое оценивание функционалов от спектральной плотности, имеющей нули. - Теория вероятн. и ее примен., 1988, т. 33 , в. 2 , с. $315-322$.

6. Гиновян М. С. Замечание о центральной предельной теореме для квадратичных форм тёплицева типа от стационарных гауссовских величин. - Изв. НАН Армении, сер. матем., 1993, т. 28, № 2, с. 78-81.

7. Ginovian M.S. On Toeplitz type quadratic functionals of stationary Gaussian process. - Probab. Theory Related Fields, 1994, v. 100, № 3, p. 395-406.

8. Giraitis L., Surgailis D. A central limit theorem for quadratic forms in strongly dependent linear variables and its application to asymptotical normality of Whittle's estimate. - Probab. Theory Related Fields, 1990, v. 86, № 1, p. 87-104.

9. Гренандер У., Сегё Г. Тёплицевы формы и их приложения. М.: ИЛ, 1961, 308 с.

10. Hasminskii R. Z., Ibragimov I. A. Asymptotically efficient nonparametric estimation of functionals of a spectral density function. - Probab. Theory Related Fields, 1986, v. 73 , № 3 , p. $447-461$.

11. Ибрагимов И. А. Об оценке спектральной функции стационарного гауссовского процесса. - Теория вероятн. и ее примен., 1963, т. 8, в. 4, с. 391-430.

12. Rosenblatt $M$. Asymptotic behavior of eigenvalues of Toeplitz forms. - J. Math. Mech., 1962, v. 11, №6, p. 941-949.

13. Сенета E. Правильно меняющиеся функции. М.: Наука, 1976, 141 с.

14. Terrin N., Taqqu M.S. A noncentral limit theorem for quadratic forms of Gaussian stationary sequences. - J. Theoret. Probab., 1990, v. 3, № 3, p. 449-475.

15. Terrin N., Taqqu M.S. Convergence in distribution of sums of bivariate Appell polynomials with long-range dependence. - Probab. Theory Related Fields, 1991, v. 90, № 1 , p. 57-81.

16. Terrin N., Taqqu M.S. Convergence to a Gaussian limit as the normalization exponent tends to $\frac{1}{2}$. - Statist. Probab. Lett., 1991, v. 11, № 5, p. 419-427.

17. Taniguchi $M$. Berry-Esseen theorems for quadratic forms of Gaussian stationary processes. - Probab. Theory Related Fields, 1986, v. 72, № 2, p. 185-194.

18. Taniguchi M., Kakizawa Y. Asymptotic Theory of Statistical Inference for Time Series. New York: Springer-Verlag, 2000, 661 p. 